\title{
Prevalence, Causes, Treatment and the Role of Antioxidants in Ischemic Brain Stroke Diseases: A Review
}

\author{
Wasi Ullah khan, Rahmat Ali Khan", Mushtaq Ahmad, Latif ullah Khan, M. Waqas Khan, \\ Samran Ahmad Durrani, Wasim Ahmed
}

Department of Biotechnology, Faculty of Biological Sciences University of Science \& Technology, Bannu KPK-Pakistan

Email address:

rahmatgul_81@yahoo.com (R. A. Khan)

\section{To cite this article:}

Wasi Ullah khan, Rahmat Ali Khan, Mushtaq Ahmad, Latif ullah Khan, M. Waqas Khan, Samran Ahmad Durrani, Wasim Ahmed. Prevalence, Causes, Treatment and the Role of Antioxidants in Ischemic Brain Stroke Diseases: A Review. American Journal of Biomedical and Life Sciences. Special Issue: Phytochemical and Pharmacological Study of Medicinal Plants. Vol. 3, No. 2-1, 2015, pp. 29-32.

doi: $10.11648 /$ j.ajbls.s.2015030201.15

\begin{abstract}
This literature review reveals that in the ischemic stroke the blood clot occur in the brain due to which the arteries blocks and stop the blood flow. There are main two types of ischemic stroke one is thrombolic and the other one is embolic. The prevalence of ischemic stroke is about $85 \%$ in the U.S while in Pakistan as such no true incidence of stroke was determined but estimated annual rate is $250 / 100,000$ translating to 350,000 new cases every year. The ischemic stroke is more than haemoregic stroke. Approximately 70 percent of patients have ischemic stroke. In the ischemic stroke there are many different types of risk factors or causes are involved due to which the ischemic stroke may cause e.g. oxidative stress, blood pressure, obesity, sex, family history etc. Many ROS are generated and may cause the oxidative stress due to which the brain is damaged and may cause the ischemic stroke. These risk factors may control through proper medications, regular exercise and the intake of many dietary antioxidants. In this type of disease the many different types of antioxidants e.g. (Bilirubin and Glutathione, Tocopherols, Dietary Vitamin E, Antioxidant Vitamin Supplements, Dietary Vitamin C) are also involved which scavenge the free radicals and reduce the chance of oxidative stress and thus the brain prevented from the damage.
\end{abstract}

Keywords: Ischemic Stroke, ROS, Oxidative Stress, Antioxidants

\section{Introduction}

Ischemic stroke is a type of brain stroke that is caused by a blood clot in a brain is called an ischemic stroke .In everyday life, blood clotting is not harmful but they are beneficial. Because when you are bleeding from a wound, the blood clots may work to slow and finally stop the bleeding. But in the case of a stroke, the blood clots are very dangerous and harmful because due to clot they can block arteries and stop blood flow. This is also called cerebrovascular disease (CVD) which is the third leading cause of death in the U.S [1]. Approximately $50 \%$ of CVD patients $\geq 65$ years of age are hemiparesis, while $30 \%$ cannot walk without support, the $19 \%$ had aphasia, while $35 \%$ are those which have depressive symptoms 6 months after ischemic stroke [2]. Repeated events are particularly dangerous and very harmful and very costly [3,4]. Approximately $87 \%$ of strokes are ischemic [1]. In Pakistan, it is estimated or expected that every year 350,000 new strokes occur in the whole country $[5,6]$.
Approximately 70 percent of patients have ischemic stroke. Ischemic stroke rates higher than haemoregic stroke.

\subsection{Types of Ischemic Stroke}

There are two main types of ischemic stroke.

A. Embolic strokes

B. Thrombotic strokes

\subsubsection{Embolic Strokes}

During or at some stage of embolic stroke, a clot travels from a secluded source and lodges in cerebral vessels. Micro emboli can break away from a sclerosis plaque in the carotid artery or from cardiac sources such as atrial fibrillation, patent foramen ovale, or a hypo kinetic left ventricle [7]. Emboli also in the form of blood, sometime may appear in the form of fat, or air can occur during surgical procedures, while most commonly during cardiac surgery, but also after 
long bone surgeries [8].

Emboli may arise or appear from the heart, the extra cranial arteries, which including the aortic arch or, rarely, the right-sided circulation (paradoxical emboli) with later passage through a patent foramen ovale [9]. The main Sources of cardiogenic emboli include the following:

- Valvular thrombi

- Mural thrombi

- Atrial myxoma

\subsubsection{Thrombotic Strokes}

In the thrombotic strokes the blood flow is blocked to the brain. In this type of stroke, thrombogenic factors may include injury to and loss of endothelial cells; this loss shows or expose the sub endothelium and due to sub endothelium platelet activation may occur, activation of the clotting cascade, inhibition of fibrinolysis, and blood stasis. Thrombotic strokes are generally originate or may arise on ruptured atherosclerotic plaques. Arterial stenosis can cause unstable blood flow, which can promote or proceed thrombus formation; atherosclerosis (i.e ulcerated plaques); and platelet adherence. All cause the formation of blood clots that either embolize or occlude the artery.

The Intracranial atherosclerosis also one of the cause of thrombotic stroke in patients with widespread atherosclerosis. While in some other patients especially younger ones, other causes should be measured, including the following $[8,10]$.

- Hypercoagulable states (e.g., protein C deficiency, protein S deficiency, pregnancy etc)

- Sickle cell disease

- Fibro muscular dysplasia

- Arterial dissections

- Vasoconstriction associated with substance abuse (eg, cocaine, amphetamines)

\subsection{Etiology}

As a result of Ischemic strokes blood flow decreases or stop, such as extra cranial or intracranial thrombotic embolism, thrombosis in situ, or relative hypo perfusion. Decreasing the blood flow the neurons stop functioning.

\subsection{Risk Factors}

There are different types of risk factors are involved for ischemic stroke. In this risk factors some of the modifiable and some are non-modifiable conditions. Identification of risk factors in each patient can reveal clues to the cause of the stroke and the most appropriate treatment and secondary prevention plan.

\subsection{Non-Modifiable Risk Factors}

The following are the non-modifiable risk factors for ischemic stroke (although there are likely many others) [11]

- Sex

- Race

- Ethnicity

- History of migraine headaches
- Fibro muscular dysplasia

- Heredity: Family history of stroke or transient ischemic attacks (TIAs)

\subsection{Modifiable Risk Factors}

There are many Modifiable risk factors also involved for ischemic srtoke. [12]

- Hypertension (the most important)

- Diabetes mellitus

- Cardiac disease:

- TIAs

- Carotid stenosis

- Hyperhomocystinemia

- Lifestyle issues: Excessive alcohol intake, tobacco use, illicit drug use, physical inactivity[13]

- Obesity

- Oral contraceptive use/postmenopausal hormone use

- Sickle cell disease

\subsection{Controlling of Risk Factors}

We also control this risk factor through proper diet, exercise, proper medications and also we decrease the risk factor to stop smoking.

\subsection{How Are Ischemic Strokes Diagnosed}

When someone has shown symptoms of a stroke or a TIA (transient ischemic attack), a doctor will collect information and make a diagnosis. He or she will review the events that have occurred and will.

- search out a medical history

- perform a physical and neurological examination

- there are many different types of laboratory (blood) tests done get a CT or MRI scan of the patient .

- study the results of other diagnostic tests that might be needed. [14]

\subsection{Role of Oxidative Stress in Ischemic Stroke}

During cerebral ischemia/reperfusion, considerable amounts of oxygen free radicals (oxidants) are generated and brain damage after stroke may occur due to oxidative stress. Experimental data from laboratory animals that either overexpress (transgenic) or are deficient in (knock-out) antioxidant proteins, mainly superoxide dismutase, have provided strong confirmation of the role of oxidative stress in ischemic brain damage. In recent reports reveal that NADPH oxidase (NOX), also an important pro-oxidant enzyme, which is involved in the production of oxidants in the brain after stroke. Inhibition of NOX is neuroprotective against cerebral ischemia. The superoxide dismutase and NOX activity in the brain is a major determinant for ischemic damage/repair and that these most important anti- and prooxidant enzymes are potential endogenous molecular targets for stroke therapy.[15] 


\subsection{Role of Different Antioxidants in Ischemic Brain Stroke Disease}

Antioxidants are molecules which scavenge the free radicals or ROS and stop the chain reaction. The Human bodies contain or prepare the powerful antioxidant defense system that controls harmful reactions of ROS. In This system some of the antioxidants are endogenous antioxidants (e.g., albumin, bilirubin, glutathione), while some are exogenous antioxidants (e.g., tocopherols, carotenoids, vitamin C) and they also have some of the antioxidant enzymes (e.g., superoxide dismutase (SOD), catalase (CAT), glutathione peroxidase $(\mathrm{GPx}))$, which alter substrates (superoxide anion radicals and hydrogen peroxide) to less reactive forms. In the following we have discussed the role and function of some antioxidants.

\subsubsection{Albumin}

Albumin is a major antioxidant in plasma, which accounts accounting for $70 \%$ of free radical-trapping activity of serum because they have the thiol group and also high plasma concentration [16].

\subsubsection{Bilirubin and Glutathione (GSH)}

This is also have antioxidant properties in serum and may prevent or protect the LDL from oxidation. [17].

\subsubsection{Tocopherols}

These are the forms of vitamin E, which have the chainbreaking lipophilic compounds that be present in human plasma and LDL in four is oforms ( $\alpha$-tocopherol, $\gamma$ tocopherol, $\beta$-tocopherol, and $\delta$-tocopherol) [18]. The $\gamma$ tocopherol is the major form of vitamin $\mathrm{E}$ in diet, while $\alpha$ tocopherol has the highest bioavailability [19].

\subsubsection{Dietary Vitamin $E$}

Several large group studies have been investigated the effects of dietary vitamin E intake on CVD risk. [20.21.22].

\subsubsection{Antioxidant Vitamin Supplements}

There are different types of antioxidant vitamine suplements e.g vitamin $\mathrm{E}$, vitamin $\mathrm{C}$, and $\beta$-carotene may reduce CVD risk.

In last there are many different types of antioxidants are found which perform their role in the reduction of the ischemic stroke diseases.

\section{Treatment}

In the ischemic stroke disease the time is very important. Stroke patients should hospitalized within 60 minutes. The goal of treatment in to restore blood flow to the affected area of the brain as soon as possible. The different types of medicines are used for the early treatment of ischemic stroke are alteplase, aspirin, and anticoagulants. In the ischemic stroke deases the early clot lysis or dissolved with recombinant tissue plasminogen activator (rtPA) up to $3 \mathrm{~h}$ after ischemic stroke $[24,25]$. The rtPA treatment therapy is the only approved therapy for acute ischemic stroke [26].
There are many others medicines are also available which lysis the blood clot.

\subsection{Aspirin}

This type of medicines only helps to prevent new clots from developing. These agents do not dissolve clots that are already present. The doctors may use early aspirin therapy (within 48 hours of the start of stroke symptoms) for patients with ischemic stroke who are not receiving alteplase or anticoagulants. The main function of aspirin is that they protect the body from another clot.

\subsection{Anticoagulants}

They are also used as a medicine and referred to as blood thinners agents. They decreasing the formation of additional blood clots . Heparin and low molecular weight heparin are used as a anticoagulants. There are many different types of medicines are also available which perform or work against yhis deases.

\section{Conclusion}

Finally we concluded that the ischemic stroke is the very serious disease. Sometimes the patient may lead to death but if we do the proper medication, do the regular exercise and make control on the blood pressure, we may control such type of diseases.

\section{References}

[1] Brooks. M, "Stroke, a Race Against the Clock." Review Confirms. Medscape Medical News. Available at http://www.medscape.com/viewarticle/830611. Accessed September 2, 2014.

[2] Emberson. J, Lees. K.R, Lyden. P, Blackwell. L, Albers. G, Bluhmki. E, et al. "Effect of treatment delay, age, and stroke severity on the effects of intravenous thrombolysis with alteplase for acute ischaemic stroke": a meta-analysis of individual patient data from randomised trials. Lancet. Aug 52014

[3] [Guideline] Adams HP Jr, del Zoppo G, Alberts MJ, Bhatt DL, Brass L, Furlan A, et al. Guidelines for the early management of adults with ischemic stroke: a guideline from the American Heart Association/American Stroke Association Stroke Council, Clinical Cardiology Council, Cardiovascular Radiology and Intervention Council, and the Atherosclerotic Peripheral Vascular Disease and Quality of Care Outcomes in Research Interdisciplinary Working Groups: the American Academy of Neurology affirms the value of this guideline as an educational tool for neurologists. Stroke. May 2007;38(5):1655-711. [Medline].

[4] Adams HP Jr, Davis PH, Leira EC, Chang KC, Bendixen BH, Clarke WR, et al. Baseline NIH Stroke Scale score strongly predicts outcome after stroke: A report of the Trial Org 10172 in Acute Stroke Treatment (TOAST). Neurology. Jul 13 1999;53(1):126-31. [Medline].

[5] Khealani BA, Wasay M. The burden of stroke criteria used in previous stroke thrombolysis trials, in Pakistan. Int J Stroke 2008;2:1-4. 
[6] Kamal AK, Khealani BA, Ansari SA, Afridiv Early is chemic stroke presentation in Pakistan. Can $J$ Neurol Sci. 2009;36:181-6.

[7] Hickey, J. V. (2003). The clinical practice of neurological and neurosurgical nursing (5th ed.). Philadelphia: Lippincott, Williams \& Wilkins.

[8] Warlow, C., Dennis, M., van Gign, J., Hankey, G. J., Sandercock, P., Bamford, J., et al. (2001). Stroke: A practical guide to management (2nd ed.). London: Blackwell Science.

[9] Arboix A, Alio J. Acute cardioembolic cerebral infarction: answers to clinical questions. Curr Cardiol Rev. Feb 2012;8(1):54-67. [Medline].

[10] Adams H, Adams R, Del Zoppo G, Goldstein LB. Guidelines for the early management of patients with ischemic stroke: 2005 guidelines update a scientific statement from the Stroke Council of the American Heart Association/American Stroke Association. Stroke. Apr 2005;36(4):916-23. [Medline].

[11] Brooks M. Migraine Linked to Double Risk for Silent Stroke. Medscape Medical News. Available at http://www.medscape.com/viewarticle/825451. Accessed May 27, 2014.

[12] Guideline] Goldstein LB, Bushnell CD, Adams RJ, Appel LJ, Braun LT, Chaturvedi S, et al. Guidelines for the primary prevention of stroke: a guideline for healthcare professionals from the American Heart Association/American Stroke Association. Stroke. Feb 2011;42(2):517-84. [Medline]

[13] Kurl S, Laukkanen JA, Rauramaa R, Lakka TA, Sivenius J, Salonen JT. Cardiorespiratory fitness and the risk for stroke in men. Arch Intern Med. Jul 28 2003;163(14):1682-8. [Medline]

[14] Department of American Heart association and Stroke association.

[15] Hai Chen,et all ,Departments of Neurosurgery and Neurology and Neurological Sciences and Program in Neurosciences, Stanford University School of Medicine, Stanford, California.

[16] Roche M., Rondeau P., Singh N.R., Tarnus E., Bourdon E. The antioxidant properties of serumalbumin. FEBS. Lett. 2008;582:1783-1787.doi: $\quad$ 10.1016/j.febslet.2008.04.057. [PubMed] [Cross Ref]

[17] Sedlak T.W., Saleh M., Higginson D.S., Paul B.D., Juluri K.R., Snyder S.H. Bilirubin and glutathione have complementary antioxidant and cytoprotective roles. Proc. Natl. Acad. Sci. USA. 2009;106:5171-5176.[PMC free article] [PubMed]
[18] Burton G.W., Joyce A., Ingold K.U. Is vitamin E the only lipid-soluble, chain-breaking antioxidant in human blood plasma and erythrocyte membranes? Arch. Biochem. Biophys. 1983;221: 281-290. doi: 10.1016/0003-9861(83)90145-5. [PubMed] [Cross Ref]

[19] Traber M.G. Vitamin E regulatory mechanisms. Annu. Rev. Nutr. 2007;27:347-362. doi: 10.1146/annurev.nutr.27.061406.093819. [PubMed] [Cross Ref]

[20] Stampfer M.J., Hennekens C.H., Manson J.E., Colditz G.A., Rosner B., Willett W.C. Vitamin E consumption and the risk of coronary disease in women. N. Engl. J. Med. 1993;328:1444-1449. doi: 10.1056/NEJM199305203282003. [PubMed] [Cross Ref]

[21] Rimm E.B., Stampfer M.J., Ascherio A., Giovannucci E., Colditz G.A., Willett W.C. Vitamin E consumption and the risk of coronary heart disease in men. N. Engl. J. Med. 1993;328:1450-1456. doi: 10.1056/NEJM199305203282004. [PubMed] [Cross Ref]

[22] Kushi L.H., Folsom A.R., Prineas R.J., Mink P.J., Wu Y., Bostick R.M. Dietary antioxidant vitamins and death from coronary heart disease in postmenopausal women. N. Engl. J. Med. 1996;334:1156-1162. doi: 10.1056/NEJM199605023341803. [PubMed] [Cross Ref]

[23] Osganian S.K., Stampfer M.J., Rimm E., Spiegelman D., Hu F.B., Manson J.E., Willett W.C. Vitamin $\mathrm{C}$ and risk of coronary heart disease in women. J. Am. Coll. Cardiol. 2003;42:246-252. doi: 10.1016/S0735-1097(03)00575-8. [PubMed] [Cross Ref]

[24] [No authors listed] Tissue plasminogen activator for acute ischemic Stroke. The National Institute of Neurological Disorders and Stroke rt-PA Stroke Study Group. N. Engl. J. Med. 333,

[25] 1581-1587 (1995).

[26] Kwiatkowski, T. G. et al. Effects of tissue plasminogen activator for acute ischemic stroke at one year. National Institute of Neurological Disorders and Stroke Recombinant Tissue Plasminogen Activator Stroke Study Group. N. Engl. J. Med. 340, 1781-1787 (1999).

[27] Grotta, J. \& Marler, J. Intravenous rt-PA: a tenth anniversary reflection. Surg. Neurol. 68 (Suppl. 1), S12-S16 (2007). 